\title{
STRONG REGULARITY AT NONPEAK POINTS
}

\section{JAMES LI-MING WANG}

ABSTRACT. We construct a uniform algebra which is strongly regular at a nonpeak point.

1. Let $A$ be a uniform algebra and $x$ be a point in $M(A)$, the maximal ideal space of $A$. We denote by $M_{x}$ the maximal ideal at $x$ and by $N_{x}$ the ideal of functions vanishing in a neighborhood of $x$. We say that $A$ is strongly regular at $x$ if $N_{x}$ is uniformly dense in $M_{x}$. It has been conjectured that if $A$ is strongly regular at $x$, then $x$ is a peak point. In a recent paper, Chalice [2] constructed a nontrivial uniform algebra which is strongly regular at every point in a dense subset $E$ of $M(A)$ while each point of $E$ is a peak point. In this note, we construct a uniform algebra which is strongly regular at a nonpeak point, and thus give a negative answer to this conjecture.

2. Let $X$ be a compact subset of the plane. We denote by $R(X)$ the uniform closure of all the (restrictions to $X$ of) rational functions having no poles on $X$. We note that $R(X)$ is strongly regular at a point $x$ in $X$ if and only if the function $z-x$ belongs to $\bar{N}_{x}$, the uniform closure of $N_{x}$, since the ideal $\{(z-x) f: f \in R(X)\}$ is uniformly dense in $M_{x}$.

Throughout this note, $\Delta(a, r)$ will denote the open disk with center $a$ and radius $r ; E^{\prime}$ will denote the complement of a set $E$.

We will need the following lemma, due to McKissick [3]:

Lemma. Let $D$ be the open unit disk. There is a sequence $\left\{a_{k}\right\}$ in $D$, $0<\left|a_{k}\right| \leq\left|a_{k+1}\right| \rightarrow 1$, such that, for any $\epsilon>0$, there is a sequence $\left\{\Delta\left(a_{k}, r_{k}\right)\right\}$ in $D$ and a sequence $\left\{f_{n}\right\}$ of rational functions with the following properties:

(1) $\Sigma_{1}^{\infty} r_{k}<\epsilon$

(2) The poles of $f_{n}$ lie in $\left\{a_{1}, \ldots, a_{n}\right\}$.

(3) $f_{n} \rightarrow F$ uniformly on $\left(\bigcup_{k} \Delta\left(a_{k}, r_{k}\right)\right)^{\prime}$ and $F \equiv 0$ on $D^{\prime}$ while $F(0)=1$.

Received by the editors May 23, 1974.

AMS (MOS) subject classifications (1970). Primary 30A82, 46J10. 
Theorem. There is a uniform algebra which is strongly regular at a nonpeak point.

Proof. We choose a sequence $\left\{\epsilon_{m}\right\}$ of positive numbers with $\Sigma \epsilon_{m}<$ $\left|a_{1}\right|$. For each fixed $m$, let $\left\{\Delta\left(a_{k}, r_{m, k}\right)\right\}_{k=1}^{\infty}$ and $\left\{f_{m, n}\right\}_{n=1}^{\infty}$ be chosen as in the Lemma so that

(1) $\sum_{k=1}^{\infty} r_{m, k}<\epsilon_{m}$.

(2) The poles of $f_{m, n}$ lie in $\left\{a_{1}, \ldots, a_{n}\right\}$.

(3) $f_{m, n} \rightarrow F_{m}$ uniformly on $\left(\bigcup_{k} \Delta\left(a_{k}, r_{m, k}\right)\right)^{\prime}$ as $n \rightarrow \infty$ and $F_{m} \equiv 0$ on $D^{\prime}$ while $F_{m}(0)=1$.

We also choose $\delta_{m}>0$ such that $|\omega|<\delta_{m}$ implies $\left|F_{m}(\omega)-1\right|<\epsilon_{m}$. Let $\left\{D_{k}^{m}\right\}_{k=1}^{\infty}$ be the images of $\left\{\Delta\left(a_{k}, r_{m, k}\right)\right\}_{k=1}^{\infty}$ under the linear fractional transformation $L_{m}: \omega \rightarrow z=\delta_{m} \epsilon_{m}\left\|F_{m}\right\|^{-1} \omega^{-1}$. We set $X=\vec{D} \backslash \bigcup_{m_{1}=1}^{\infty}\left\{D_{k}^{m}\right\}$. It is easy to compute that

$$
\sum_{m, k=1}^{\infty}\left(\text { radius of } D_{k}^{m}\right) / \mid \text { center of } D_{k}^{m}\left|=\sum_{m, k=1}^{\infty} \frac{{ }^{r}, k}{\left|a_{k}\right|}<\left(\sum \epsilon_{m}\right) /\right| a_{1} \mid<1 \text {. }
$$

Thus 0 is a nonpeak point for $R(X)$ since there exists a representing measure $\mu$ for 0 with $\mu(\{0\})=0$ (see, e.g., [1, p. 99]).

We now prove that $R(X)$ is strongly regular at 0 . If we set $g_{m}(z)=$ $z F_{m}\left(L_{m}^{-1}(z)\right)$ and $U_{m}=\left\{z:|z|<\epsilon_{m} /\left\|F_{m}\right\|\right\}$, then $g_{m} \in N_{0}$. We have $\left|g_{m}-z\right|=\left|F_{m}\left(L_{m}^{-1}(z)\right)-1\right| \cdot|z|$. This is no more than $\epsilon_{m}$ on $U_{m}^{\prime} \cap X$, and it is no more than $\left(\left\|F_{m}\right\|+1\right)\left(\epsilon_{m} /\left\|F_{m}\right\|\right)$ on $U_{m}$, which implies that $z \in \bar{N}_{0}$ (we may assume $\left\{\left\|F_{m}\right\|\right\}$ is nondecreasing), so the conclusion follows from an early remark.

\section{REFERENCES}

1. A. Browder, Introduction to function algebras, Benjamin, New York, 1969. MR 39 \#7431. $300-304$.

2. D. R. Chalice, S-algebras on sets in $C^{n}$, Proc. Amer. Math. Soc. 39 (1973),

3. R. McKissick, A nontrivial normal sup norm algebra, Bull. Amer. Math. Soc. 69 (1963), 391-395. MR 26 \#4166.

DE PARTMENT OF MATHEMATICS, BROWN UNIVERSITY, PROVIDENCE, RHODE ISLAND 02912

Current address: Department of Mathematics, University of California, Los Angeles, California 90024 